\title{
CFD Numerical Simulation of Hydrodynamics in a Rotor-Stator Reactor for Biodiesel Synthesis
}

\author{
Zhuqing Wen, Jerzy Petera \\ Faculty of Process and Environmental Engineering, Lodz University of Technology, Lodz, Poland \\ Email: jievan-0513@163.com
}

Received 11 June 2015; accepted 19 August 2015; published 26 August 2015

\begin{abstract}
In this paper a rotor-stator spinning disk reactor for intensified biodiesel synthesis is described and numerically simulated. The reactor consists of two flat disks, located coaxially and parallel to each other with a gap ranging from $0.1 \mathrm{~mm}$ to $0.2 \mathrm{~mm}$ between the disks. The upper disk is located on a rotating shaft while the lower disk is stationary. The feed liquids, triglycerides (TG) and methanol are introduced coaxially along the center line of rotating disk and stationary disk, respectively. Fluid hydrodynamics in the reactor for synthesis of biodiesel from TG and methanol in the presence of a sodium hydroxide catalyst are simulated, using convection-diffusion-reaction species transport model by the CFD software ANSYS ${ }^{\odot}$ Fluent v. 13.0. The effects of upper disk's spinning speed and gap size are evaluated.
\end{abstract}

\section{Keywords}

Rotor-Stator Reactor, Biodiesel Synthesis, ANSYS ${ }^{\odot}$ Fluent, Hydrodynamics, Numerical Simulation

\section{Introduction}

Biodiesel production involves the transesterification of oil or fat feedstock with methyl alcohol under alkaline conditions in a liquid-liquid environment. Transesterification is a liquid-liquid two phase reaction. Reaction rate can be limited by mass transfer between the oils and alcohol because they are immiscible.

Rotor-stator spinning disk reactor (SDR) is one of the process intensification technology employing high gravity fields caused by centrifugation. In some existing solutions of SDR, a high gravity field-centrifugal force is created by rotation of a disk surface on which liquid is dispersed as a thin film. When a liquid is introduced onto the disk surface at or adjacent to the spin axis, the liquid flows radially outward under the centrifugal force in the form of a thin film. At up to approximately 1000 rpm, these films are less than 100 microns thick and so offer a short diffusion path length [1]. Unsteady film surface waves on the disk surface, coupled with the shearing action of the rotating surface, ensure that micro mixing and excellent mass and heat transfer are achieved [2].

The hydrodynamics inside SDR have important effects on conversion, mass and heat transfer rates. Flow 
structures in single phase rotor-stator systems have been investigated extensively over the past decades [3], and research is still ongoing. However, direct application of the complex hydrodynamics to actual SDR for design and performance calculations is often unfeasible. Therefore a simplified engineering model of the fluid flow patterns inside rotor-stator cavities is assumed and depicted [4], the flow through a rotor-stator cavity of the SDR can be described as a combination of radial plug flow and ideally mixed regions.

In the present research, a special version of SDR developed to explore the possibility of improving the efficiency of biodiesel production is described and used, as shown in Figure 1. Although this SDR is well used in biodiesel production, it's still unclear how fluid hydrodynamics influenced the performance of SDR. In this situation the complex process can be investigated by numerical simulation using professional CFD software. ANSYS $^{\complement}$ Fluent 13.0 was chosen in the present research. Fluid hydrodynamics in the reactor for the synthesis of biodiesel were modelled using convection-diffusion-reaction multicomponent transport model. In particular the effects of upper disk’s spinning speed and gap size were investigated.

\section{Experimental Setup and Mechanism of Alkali-Catalyzed Transesterification Reaction}

The experimental setup was built and described by Zheyan Qiu and Weatherley [5], who are our collaborative partners from Department of Chemical and Petroleum Engineering, Kansas University, USA. The SDR system consisted of two parallel coaxial disks: one stationary and the other rotational with controllable rotational speed. The disks in the present research were separated by a gap range of $0.1 \mathrm{~mm}$ to $0.2 \mathrm{~mm}$. The two immiscible liquid phases were pumped axially at the centers of the stationary disk and the spinning disk, with the speed of $0.007 \mathrm{~m} \cdot \mathrm{s}^{-1}$ and $0.006 \mathrm{~m} \cdot \mathrm{s}^{-1}$, respectively. The model reaction system chosen for study was the methyl-esterification of canola oil in the presence of a sodium hydroxide catalyst to form biodiesel and glycerol. Before being pumped into SDR, sodium methoxide was prepared by dissolving sodium hydroxide in methanol. The experimental setup is shown in Figure 1.

During the transesterification of TG, there are three stepwise and reversible reactions with intermediate formation of diglycerides (DG) and monoglycerides (MG) resulting in the production of methyl esters (RCOOCH3, biodiesel) and glycerol (GL) as shown in chemical equations I-III. The overall transesterification reaction is shown as chemical equation IV [6] [7].

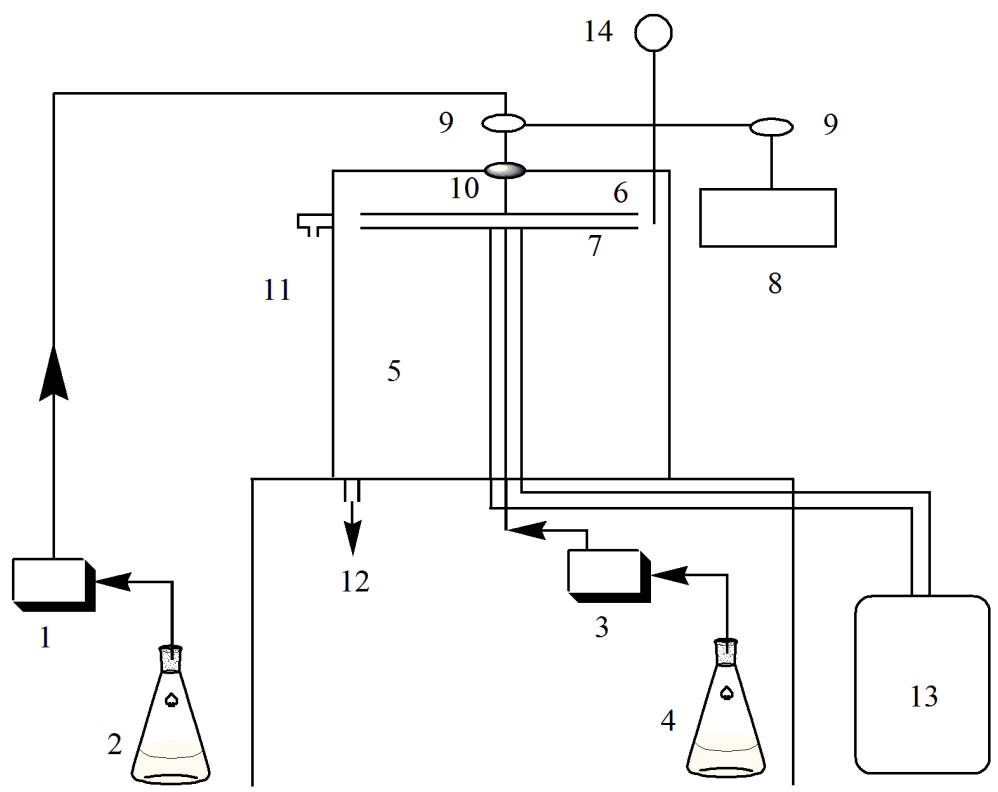

Figure 1. Experimental setup of the intensive SDR for biodiesel synthesis: (1) peristaltic pump; (2) canola oil vessel; (3) digital piston pump; (4) sodium methoxide vessel; (5) cylinder; (6) rotating disk; (7) stationary disk; (8) variable-speed DC motor; (9) pulley; (10) bearing; (11) sampling point; (12) products drainage; (13) heating circulator; (14) thermometer. 


$$
\begin{aligned}
& \mathrm{TG}+\mathrm{CH}_{3} \mathrm{OH} \stackrel{k_{1}}{\stackrel{k_{2}}{\longrightarrow}} \mathrm{DG}+\mathrm{RCOOCH}_{3} \\
& \mathrm{DG}+\mathrm{CH}_{3} \mathrm{OH} \underset{k_{4}}{\stackrel{k_{3}}{\longrightarrow}} \mathrm{MG}+\mathrm{RCOOCH}_{3} \\
& \mathrm{MG}+\mathrm{CH}_{3} \mathrm{OH} \underset{k_{6}}{\stackrel{k_{5}}{\longleftrightarrow}} \mathrm{GL}+\mathrm{RCOOCH}_{3} \\
& \mathrm{TG}+3 \mathrm{CH}_{3} \mathrm{OH} \stackrel{\text { catalyst }}{\rightleftarrows} 3 \mathrm{RCOOCH}_{3}+\mathrm{GL}
\end{aligned}
$$

\section{Simulation}

ANSYS $^{\oplus}$ Fluent v.13.0 was chosen to simulate the biodiesel synthesis process in SDR. It is important to appropriately mesh the computational domain. To obtain satisfactory mesh, it is necessary to simplify the real construction of SDR. As it can be seen from Figure 1, the region of interest is the space between spinning disk and stationary disk. Besides, this construction is axisymmetric, hence, only half of it was simulated. The computational domain is shown in Figure 2.

\subsection{Simulation Methodology}

ANSYS $^{\odot}$ Fluent predicts the local mass fractions of each species through the solution of convection-diffusionreaction equation for each species. The general form of this equation is:

$$
\partial\left(\rho Y_{i}\right) / \partial t+\nabla \cdot\left(\rho v Y_{i}\right)=-\nabla \cdot J_{i}+R_{i}+S_{i}
$$

where, $\rho$ is density of species, $Y_{i}$ is mass fraction of species $i, v$ is velocity of species, $J_{i}$ is diffusional flux of species $i, R_{i}$ is net rate of production of species $i$ by chemical reaction, $S_{i}$ is rate of creation of species $i$ by additional source.

The diffusion flux of species $i, J_{i}, i=1,2, \ldots, 5$ which arises from the corresponding concentration gradients, were defined as

$$
J_{i}=-\rho D_{i, m ?} \nabla Y_{i}
$$

For the calculation of chemical reactions, the laminar finite-rate model was used based on the Arrhenius expressions. The net source of chemical species $i$ due to reaction $R_{i}$ was computed as the sum of the Arrhenius reaction sources over the $N_{R}$ reactions that the species participate in:

$$
R_{i}=M_{w, i} \cdot \sum_{r=1}^{N_{R}} R_{i, r}^{\prime}
$$

In the present research, the kinetic rate constant and activation energy data were obtained and modified on the basis of data in Table 1.

Concerning to the last term in the general Equation (1), $S_{i}$ equals to zero because in the present research there is no additional source for species.

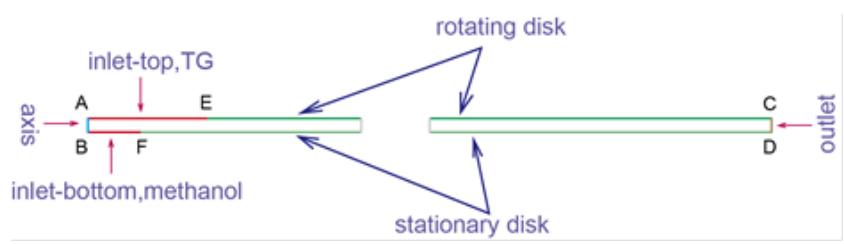

Figure 2. Simplified computational domain of SDR.

Table 1. Chemical kinetics data used and modified according to the units used in the simulation $\left(25^{\circ} \mathrm{C}\right)$ [7].

\begin{tabular}{cccc}
\hline Parameter & Reaction I (TG $\rightarrow$ DG) & Reaction II (DG $\rightarrow$ MG) & Reaction III $($ MG $\rightarrow$ GL) \\
\hline Kinetic rate constant, $k_{i}$ & $8.333 \times 10^{-4}$ & $3.583 \times 10^{-3}$ & $4.033 \times 10^{-3}$ \\
Activation energy, $E_{i}$ & $5.5 \times 10^{7}$ & $8.309 \times 10^{7}$ & $2.687 \times 10^{7}$ \\
\hline
\end{tabular}




\subsection{Modeling Assumptions and Boundary Conditions}

For the purpose of modeling and in order to simplify the calculation, initial assumptions were made as follows:

- Feedstock from inlet-top and inlet-bottom was TG and methanol, respectively. Molar ratio of methanol to TG was 6:1, and concentration of $\mathrm{NaOH}$ was $1.0 \mathrm{wt} \%$. There was no explicit occurrence of a catalyst, however the presence of the catalyst was taken into account by modification of the rate constants in the kinetic equations;

- Problem was nearly isothermal because the thermal boundary conditions assumed perfect thermal control, thus all physicochemical properties were nearly constant. In the present research, all cases were simulated under the temperature of $25^{\circ} \mathrm{C}$.

- At the start the whole domain (the gap) was filled with TG at rest and from this point the process started by injecting substrates TG and methanol.

\subsection{Available Experimental Data Dictated the Following Modeling Conditions}

- Due to the fact that canola oil, as a mixture of fatty acids, consists mainly of oleic acid, triolein $\left(\mathrm{C}_{57} \mathrm{H}_{104} \mathrm{O}_{6}\right)$ was chosen as a representation of $\mathrm{TG}$, then diolein $\left(\mathrm{C}_{39} \mathrm{H}_{72} \mathrm{O}_{5}\right)$ as a representation of DG, monoolein $\left(\mathrm{C}_{21} \mathrm{H}_{40} \mathrm{O}_{4}\right)$ as a representation of $\mathrm{MG}$, and methyl oleate $\left(\mathrm{C}_{19} \mathrm{H}_{36} \mathrm{O}_{2}\right)$ as a representation of the resulting methyl ester (biodiesel).

- The inlet velocities for TG (inlet-top) and methanol (inlet-bottom) were $0.006 \mathrm{~m} \cdot \mathrm{s}^{-1}$ and $0.007 \mathrm{~m} \cdot \mathrm{s}^{-1}$, respectively.

- The temperature was $25^{\circ} \mathrm{C}$ everywhere.

\subsection{Simulation Results}

Figure 3 below shows velocity contours inside the SDR gap. The vertical position of the picture is justified by the ANSYS ${ }^{\odot}$ Fluent technicality. When the axisymmetric geometry is modelled the direction of swirl axis is assumed to be the $\mathrm{X}$ axis and the computational domain is situated above the $\mathrm{X}$ axis, as shown in the following figures.

Figure 3 indicates that magnitude of the bulk circumferential velocity increases from bottom right to top left. Because of fast rotation, reacting mixture close to the spinning disk flows faster, and reaches the highest speed at the upper end of the reactor.

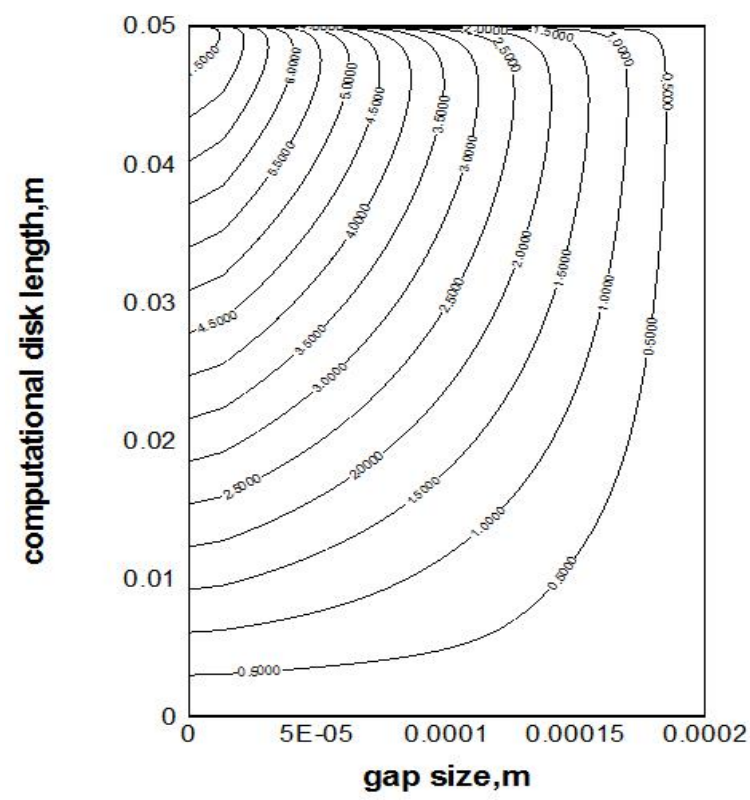

Figure 3. Contours of inter-disk circumferential velocity in $\mathrm{m} \cdot \mathrm{s}^{-1}$ (the geometry proportions are intentionally changed). 
Figure 4 presents the reverse flow zone inside the inter-disk space showing a characteristic pattern, including an interesting impinging phenomenon. Pattern such as the one shown in purple indicates where the reverse flow meets with the predominant outward stream, and then flow together outwards outlet after meeting. The boarder of this zone may move with spinning rate increase.

In the present research, the cavity inside SDR can be described by regions of radial plug flow at low radial disk positions, in combination with a single ideally mixed region at high radial positions. The radius where flow pattern changes from plug flow to ideally mixed flow, decrease as the rotational speed increases, and increases as gap size decreases.

To verify the simulation of the flow patterns inside the SDR cavity, TG conversions from experiments are used. Conversions in plug flow is larger than that in ideally mixed flow from chemical engineering point of view. That means inside SDR cavity, TG conversions should decrease as rotational speed of spinning disk increases; whereas TG conversions will increase as gap size decreases.

Figure 5 is TG conversion data from experiments attained from Qiu's work [8]. It indicates that TG conversions increase as gap size decreases and TG conversions decrease as rotational speed of spinning disk increases.

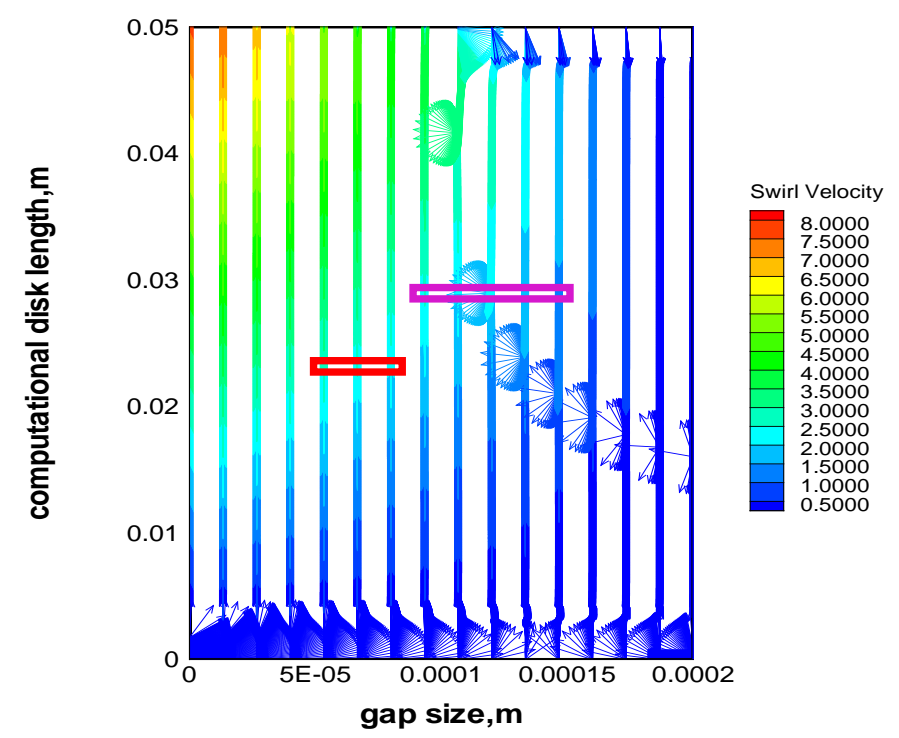

Figure 4. Overall reverse flow zone within SDR gap in form of velocity vectors (the geometry proportions are intentionally changed).

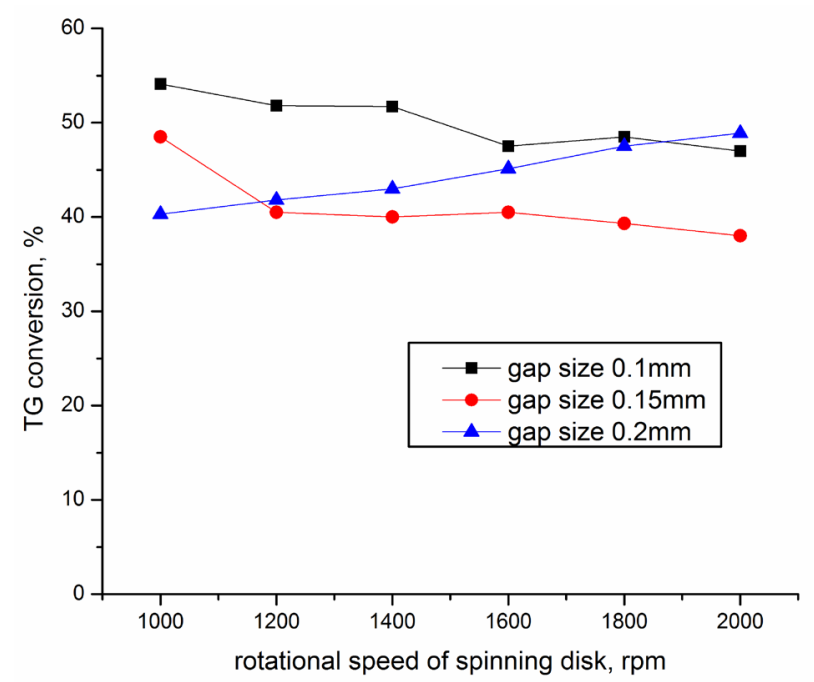

Figure 5. Effects of rotational speed of spinning disk and gap size on TG conversion. 
This is in consistent with the chemical engineering theory described above. There is an exception when gap size is $0.2 \mathrm{~mm}$, TG conversions increase as rotational speed increases, probably when gap size is $0.2 \mathrm{~mm}$, faster rotational speed results in higher shear forces and consequently enhanced the mixing of the reactants, this helps to increase TG conversion.

\section{Conclusions}

The CFD software ANSYS ${ }^{\odot}$ Fluent v.13.0 was successfully used for simulation of biodiesel synthesis from canola oil and methanol in the presence of sodium hydroxide catalyst in the two-disk spinning disk reactor. To accomplish this task the adequate model of reaction with accompanying mass transfer was formulated and expressed in terms of facilities available within ANSYS ${ }^{\odot}$ Fluent.

The hydrodynamics of reagents in SDR provided insights into the fluid flow profiles in the inter-disk gap which could be helpful to explain how the fluid hydrodynamics influenced the performance of SDR. The cavity inside SDR can be described by regions of radial plug flow at low radial disk positions, in combination with a single ideally mixed region at high radial positions as shown in Figure 4. The radius where flow pattern changes from plug flow to ideally mixed flow, decrease as the rotational speed increases, and increases as gap size decreases.

The effects of rotational speed of spinning disk and gap size on hydrodynamics of SDR are investigated. TG conversions attained from experiments were used to verify the simulation of hydrodynamics inside SDR. TG conversions decrease as rotational speed of spinning disk increases, whereas TG conversions increase as gap size decreases. This is in consistent with the chemical engineering theory. There is an exception when gap size is $0.2 \mathrm{~mm}$, TG conversions increase as rotational speed increases, probably when gap size is $0.2 \mathrm{~mm}$, faster rotational speed results in higher shear forces and consequently enhanced the mixing of the reactants, this helps to increase TG conversion. More simulations will be conducted to investigate factors affecting hydrodynamics inside SDR.

\section{References}

[1] Stankiewicz, A.I. and Moulijn, J.A. (2000) Process Intensification: Transforming Chemical Engineering. Chemical Engineering Progress, 96, 22-34.

[2] Jachuck, R. (1995) Process Intensification for Responsive Processing. Trans IChemE, 80, 233-238. http://dx.doi.org/10.1205/026387602753581980

[3] Owen, J.M. and Rogers, R.H. (1989) Flow and Heat Transfer in Rotating-Disc Systems, Rotor-Stator Systems in Engineering Design Series, 1, John Wiley \& Sons, Inc.

[4] de Beer, M.M., Keurentjes, J.T.F., Schouten, J.C. and van der Schaaf, J. (2014) Engineering Model for Single-Phase Flow in a Multistage Rotor-Stator Spinning Disc Reactor. Chemical Engineering Journal, 242, 53-61. http://dx.doi.org/10.1016/j.cej.2013.12.052

[5] Qiu, Z.Y., Petera, J. and Weatherley, L.R. (2012) Biodiesel Synthesis in an Intensified Spinning Disk Reactor. Chemical Engineering Journal, 210, 597-609. http://dx.doi.org/10.1016/j.cej.2012.08.058

[6] Freedman, B., Butterfield, R.O. and Pryde, E.H. (1994) Transesterification Kinetics of Soybean Oil. Journal of the American Oil Chemists' Society, 63, 1375-1380. http://dx.doi.org/10.1007/BF02679606

[7] Noureddini, H. and Zhu, D. (1997) Kinetics of Transesterification of Soybean Oil. Journal of the American Oil Chemists' Society, 74, 1457-1463. http://dx.doi.org/10.1007/s11746-997-0254-2

[8] Qiu, Z.Y. (2010) Intensification of Liquid-Liquid Contacting Processes. Ph.D. Thesis, University of Kansas, USA. 